\section{Salvage-Radiotherapie beim Prostatakarzinom}

\author{
Welche Strahlendosis ist bei der Salvage-Radiotherapie des Prostatakarzi- \\ noms nach Prostatektomie notwendig, um ein biochemisches Rezidiv zuver- \\ lässig zu verhindern? Diese Frage war Gegenstand einer Beobachtungsstudie.
}

A ufgenommen wurden 2.460 erwachsene Patienten an neun US-amerikanischen Zentren, die sich wegen eines Prostatakarzinoms (Stadium II/III) einer radikalen Prostatektomie und Lymphadenektomie unterzogen hatten und wegen wieder ansteigender PSA-Werte vor einer Salvage-Radiotherapie (RT) standen. Primärer Endpunkt war ein biochemisches Rezidiv nach RT, sekundärer Endpunkt Fernmetastasierung. Drei Therapiegruppen wurden definiert: < 66,0 Gy, 66,0-69,9 Gy und $\geq 70,0$ Gy.

Ausgewertet wurden die Daten von 1.108 Patienten. Die mediane Zeit von der radikalen Prostatektomie bis zur Salvage-RT betrug 19,3 Monate, die mediane Nachbeobachtungszeit 65,2 Monate. Im Beobachtungszeitraum zeigte sich ein Trend zu höheren Strahlendosen von mindestens 70 Gy, die im Zeitraum von 1985 bis $19991 \%$ der Patienten erhalten hatte, danach $27 \%$.

Die kumulative Inzidenz eines biochemischen Rezidivs nach Salvage-RT betrug nach fünf Jahren $36,5 \%$, nach zehn Jahren 50,2\%. Ein hoher Gleasonscore, hohe PSA-Werte vor SalvageRT und eine Invasion der Samenblasen (T3b) waren mit ungünstiger Prognose assoziiert.

Höhere Strahlendosen senkten das Risiko für ein biochemisches Rezidiv um 26-35\%. So waren beispielsweise $61 \%$ der Patienten, die mindestens 70 Gy erhalten hatten, im zehnten Jahr noch rezidivfrei, verglichen mit $43 \%$ derer, die weniger als 66 Gy erhalten hatten. Zwischen den Gruppen mit 66,0-69,9 Gy und $\geq 70,0$ Gy gab es keinen Unterschied.
Die kumulative Inzidenz distaler Metastasen betrug nach zehn Jahren 12,4\%. Hier waren ein hoher Gleasonscore, hohe PSA-Werte vor Salvage-RT und ein Stadium T3b mit einer ungünstigeren Prognose assoziiert. Mehr als ein Viertel der Patienten mit einem Gleasonscore von $8-10$ oder Stadium T3b entwickelte innerhalb von zehn Jahren Fernmetastasen. Eine Verzögerung der Salvage-RT bis zu PSA-Werten von mehr als 1,0-2,0 $\mathrm{ng} / \mathrm{ml}$ war mit einem dreifach höheren Risiko für Fernmetastasen assoziiert.

Fazit: In der vorliegenden Studie war kein Unterschied bezüglich biochemischer Rezidive zwischen Strahlendosen von 66 und $\geq 70$ Gy bei der Salvage-RT des Prostatakarzinoms nach Prostatektomie festzustellen. Dosen < 66 Gy waren mit einer ungünstigeren Prognose assoziiert. Kathrin von Kieseritzky

Pisansky TM et al. Salvage Radiotherapy Dose Response for Biochemical Failure of Prostate Cancer after Prostatectomy - A Multi-Institutional Observational Study. Int J Radiat Oncol Biol Phys. 2016; 96(5): 1046-53.

\section{Viszerale Metastasierung beim Prostatakarzinom}

\author{
In der AFFIRM-Studie verbesserte der Androgenrezeptorinhibitor (ARI) \\ Enzalutamid beim metastasierten kastrationsresistenten Prostatakarzinom \\ (mCRPC) das Überleben signifikant. Nachträglich wurde untersucht, wie \\ sich die Substanz bei viszeralen Metastasen auswirkt.
}

$E^{n-m i n}$ ntwickeln Patienten mit metastasiertem Prostatakarzinom eine Kastrationsresistenz, so ist die Prognose ungünstig - mit medianen Überlebenszeiten unter den derzeit verfügbaren Therapien von weniger als drei Jahren. Besonders ungünstig ist die Prognose bei viszeralen Metastasen, die bei 22-30\% der metastasierten Tumoren vorliegen.

In der jetzt vorliegenden Post-hocAnalyse wurden die Daten der Phase-IIIStudie AFFIRM ausgewertet, die Patienten mit Leber- und/oder Lungenmetastasen zum Zeitpunkt der Erstdiagnose einschloss. Unter Berücksichtigung des ECOG-Performancestatus (0-1 vs. 2) und des mittleren Schmerzscores ( $0-4$ vs. $\geq 4$ ) waren die insgesamt 1.199 Teilnehmer im Verhältnis 2:1 randomisiert mit
$160 \mathrm{mg} / \mathrm{d}$ Enzalutamid $(\mathrm{n}=800)$ oder Placebo $(n=399)$ behandelt worden. 278 $(23,2 \%)$ von ihnen hatten bei Erstdiagnose Leber- und/oder Lungenfiliae.

Patienten mit Lebermetastasen wiesen generell ungünstigere prognostische Faktoren auf als die mit nicht viszeralen beziehungsweise Lungenmetastasen. Doch sie profitierten von der Therapie mit Enzalutamid: Im Vergleich zu Placebo ergab sich unter Enzalutamid ein geringeres Risiko für radiologisch nachweisbare Progression (Hazard Ratio [HR]: 0,645), ein besseres 12-MonatsGesamtüberleben (OS; 37,7 vs. 20,6\%) und ein verbessertes radiologisch progressionsfreies Überleben (PFS; 11,6 vs. 3,0\%) sowie eine höhere PSA-Ansprechrate $(35,1$ vs. $4,8 \%)$.
Auch die Patienten mit Lungenmetastasen profitierten von Enzalutamid. Sie hatten gegenüber den mit Placebo Behandelten ein verbessertes medianes OS (HR: 0,848), ein substanziell geringeres Risiko für radiologisch nachweisbare Progression (HR: 0,386), ein verbessertes 12 -Monats-OS (65,1 vs. $55,3 \%)$ und ein verbessertes radiologisches PFS (30,9 vs. $8,2 \%$ ), außerdem eine verlängerte Zeit bis zur PSA-Progression (HR: 0,358) und eine bessere PSA-Ansprechrate (52,1 vs. $4,9 \%)$. Verglichen mit den Patienten mit nicht viszeralen Metastasen entwickelten die Patienten mit viszeralen Metastasen nicht mehr therapiebedingte Nebenwirkungen.

Fazit: Patienten mit mCRPC und Lungen- oder Lebermetastasen profitieren von der Therapie mit dem ARI Enzalutamid.

Kathrin von Kieseritzky

Loriot $Y$ et al. Enzalutamide in Castration-Resistant Prostate Cancer Patients With Visceral Disease in the Liver and/or Lung: Outcomes From the Randomized Controlled Phase 3 AFFIRM Trial. Cancer. 2017; 123(2): 253-62. 